\title{
Analysis of the Mental Health of Urban Migrant Children Based on Cloud Computing and Data Mining Algorithm Models
}

\author{
Juan Li iD \\ Shandong Management University, School of Arts, Jinan, Shandong 250357, China \\ Correspondence should be addressed to Juan Li; lijuan_smu@163.com
}

Received 19 August 2021; Accepted 3 September 2021; Published 16 September 2021

Academic Editor: Punit Gupta

Copyright (C) 2021 Juan Li. This is an open access article distributed under the Creative Commons Attribution License, which permits unrestricted use, distribution, and reproduction in any medium, provided the original work is properly cited.

\begin{abstract}
With the rapid development of internet technology, the amount of data generated is also increasing day by day. As a kind of distributed computing, cloud computing has been widely used in the analysis of massive data. With the development of China's economic construction, the integration of urban and rural areas is constantly improving, and the migrant children in the city are also focused on. After moving into the city, migrant children not only face the pressure from the society but also face the pressure from life, which inevitably affects the physical and mental health of urban migrant children. The education of urban migrant children is also a focus that needs attention. How to integrate into the education environment of urbanization and adjust the learning pressure in the process of education is also worthy of our attention. Therefore, this article analyzes the current status of urban migrant children's mental health based on cloud computing and data mining algorithm models. Based on the current research status of urban migrant children and the standards of mental health, this paper conducts a survey of middle and high school students in a certain city through questionnaires, then builds a data mining algorithm model to analyze the survey data, and explores the differences in the grades of students' social identity and the differences in mental health between migrant children and urban children. According to the survey, most of the psychological performances of urban migrant children are very vague. At the same time, there are also some phenomena such as poor adaptability, bad mood, and inferiority complex. During the study period, there are situations such as unwilling to communicate with others, weariness, sensitivity, anxiety, and hostility. The overall incidence of the situation is relatively high in big cities, while the situation of urban children is relatively small.
\end{abstract}

\section{Introduction}

Cloud computing is a kind of virtualization technology. In addition to powerful computing power and scalability, it also has the characteristics of high flexibility, high reliability, and on-demand deployment. In recent years, the rapid development of information technology has also promoted the development of cloud computing by leaps and bounds. In scientific research, building algorithm models based on cloud computing can greatly improve research efficiency $[1,2]$. In the process of my country's rapid transformation from the traditional economic development model, labor from rural areas has gradually poured into big cities, and most of them are in the family model, bringing young children to the city. According to the data of the sixth national census, in the previous 10 years, the floating population in the city increased greatly, compared with the fifth national census, and there is a very obvious growth trend $[3,4]$. Therefore, migrant children have become a huge group in the city $[5,6]$.

Because there is a big difference between the daily life of modern urban people and that of rural people in China, mobile children, as the second generation of immigrants, passively flow into the city. In daily life, learning, social environment adaptation, and other aspects, mobile children are constantly adjusting their own psychological environment, but in this process, they will experience some confusion, frustration, and loss. Because of the change of the education mode and the influence of bad family environment, it is easy to cause poor communication with peers under the trend of communication and psychological state $[7,8]$. In recent years, the mental health problems of migrant 
children in China's cities have gradually been concerned. Some migrant children are in puberty, which is also out of the rebellious stage. In the face of so many puzzles at this critical moment, whether they can sort out their emotional and psychological problems, bear the pressure brought by the mobile life, and adapt to the environment will have a great impact on the future development and mental health of migrant children. The formation of personality has an important impact $[9,10]$.

The purpose of this article is to study the mental health of urban migrant children, conduct a questionnaire survey of middle and high school students in a certain city, and then establish a data mining algorithm model in a cloud computing environment, so as to analyze the survey data and differentiate the students from their grades. Look at the changes in the psychological status of urban migrant children $[11,12]$.

\section{Data Mining Algorithm Model Construction Based on Cloud Computing}

2.1. Cloud Computing Platform. Cloud computing platforms can be divided into three categories: cloud storage platforms that focus on data storage, cloud computing platforms that focus on data processing, and comprehensive cloud computing platforms that take into account both computing and data storage and processing $[13,14]$.

As a leading cloud service provider in China, Alibaba Cloud has high stability and practicability and has been widely recognized by users. Compared with international competitors, Alibaba Cloud has a relatively strong price advantage with almost the same computing power [15]. Therefore, this article chooses Alibaba Cloud as the cloud computing platform for research.

2.2. K-Means Clustering Algorithm Model. The number of migrant children in cities is increasing year by year. Due to many reasons such as environmental inadaptability, their mental health problems deserve our attention [16]. Conducting research on urban migrant children to find out the factors that cause their mental health problems can provide parents, teachers, and functional departments with a theoretical basis for solving their psychological problems, so as to better protect this group and enable urban migrant children to grow up healthily [17]. Kmeans algorithm is a classic clustering algorithm for unsupervised learning, which has the advantages of simple thinking, good effect, and easy implementation. It is scientific and practical to analyze the mental health of urban migrant children through the $K$-means algorithm model $[18,19]$.

\section{(1) Algorithm idea:}

Divide $n$ samples into $k$ clusters, and then according to the distance formula or other similarity calculation formulas, the other points in the sample are divided into the nearest cluster; then, calculate the average of all objects in the cluster as the new center point. Iterate repeatedly until the objective function converges.

(2) Algorithm definition:

$$
\begin{aligned}
P & =\left\{p_{1}, p_{2} \ldots, p_{n}\right\} . \\
f(p i, p j) & =\sqrt{\left(p_{i 1}-p_{j 2}\right)^{2}+\cdots+\left(p_{i n}-p_{j n}\right)^{2}} . \\
\varepsilon_{i} & =\frac{1}{n} \sum_{p \in w_{i}} p . \\
F & =\sum_{i=1}^{z} \sum_{p \in w_{i}}\left|p-n_{i}\right|^{2} .
\end{aligned}
$$

(3) Algorithm process:

Selection of the $k$ value:

In the traditional $K$-means algorithm, the number of clusters $k$ is required to be determined in advance, but in practice, it is often difficult to determine the value of $k$ due to the large amount of data and lack of experience. If the value of $k$ is selected too small, it will result in data objects to vary greatly in the same cluster. If the value of $k$ is selected too large, the difference between different clusters will be small. At the same time, improper selection of the $k$ value will also make the final clustering result fall into the local optimum. This paper selects 10 factors that can reflect the mental health of urban migrant children, such as obsessivecompulsive disorder, depression, and anxiety, as a clustered dataset.

Selection of cluster center $c$ :

The steps of selecting cluster centers: first, calculate the maximum value $a 1$ and minimum value $b 1$ in the sample set, and record where they appear, and then calculate the average rounding to get the cluster center $c 1$. Then, remove the maximum value $a 1$ and minimum value $b 1$, select the approximate maximum value $a 2$ and minimum value $b 2$ to obtain the cluster center $c 2$, and so on; finally, $P=\left\{c_{1} \oplus c_{2} \oplus \cdots \oplus c_{n}\right\} / n$.

Calculate the distance between the sample and the center: calculate the distance between the sample $P=\left\{p_{1}, p_{2} \ldots, p_{n}\right\}$ and the center $c$, and divide $p_{i}$ closest to the center $c$ into the corresponding clusters.

(4) Model establishment:

(a) First, enter the survey data of urban migrant children's mental health problems, check the validity of the data, and normalize the data to ensure the accuracy and reliability of the data. The normalization formula is as follows:

$$
H=\frac{H i-H \min }{H \max -H \min } .
$$


(b) Classify the data to ensure that the classified data have strong similarity.

(c) Add the classified 10 groups of data to LSTM for training, and use the powerful time-series feature extraction ability of the LSTM neural network to obtain the feature data of urban migrant children's mental health.

(d) Use the improved $K$-means algorithm to perform clustering and output the classification results.

Definition 1. Define the dataset that needs to be clustered, as shown in the following formula:

Definition 2. (similarity calculation formula). Here, the Euclidean distance formula is selected as the similarity calculation formula, as shown in the following:

Definition 3. Cluster center point, as shown in the following formula:

Definition 4. Convergence conditions, as shown in the following formula:

\section{Survey on the Mental Health of Urban Migrant Children Based on Cloud Computing and Data Mining Algorithms}

This article uses a questionnaire survey method to conduct research. The questionnaire survey method mainly refers to a scientific exploratory research method that is generally used to collect information by publishing questions to students in writing. The researcher lists the questions that need to be discussed, fills in the content in the form of face-to-face answers or follow-up interviews, and then collects, sorts out, and comprehensively analyzes the questionnaire to obtain a large amount of data which are closely related to this question and information.

3.1. Research Purpose. It is of great significance to promote the development and improvement of the mental health to urban migrant children. Under the tense pace of life in the city, different children will also face different psychological pressures, which will change children's cognition. How to deal with the possible problems of peer discrimination, inferiority complex, and communication difficulties between families is particularly important. These problems also have a great influence on the formation of children's future personality. From the perspective of social work, it is the most important work to improve the mental health of migrant children.

\subsection{Questionnaire Design}

3.2.1. Research Object. According to the survey of two schools in a city, this paper randomly selected the reports of two different grades in junior high school and senior high school. According to the reports of these 352 students, the questionnaire is made. After the link was generated, the head teachers shared it with the class group, and the students filled in it anonymously. A total of 330 valid questionnaires were submitted by the two grades; there were 105 questionnaires for migrant children and 95 questionnaires for urban children; there were 65 questionnaires for migrant children in senior one and 64 questionnaires for urban children.

\subsubsection{Research Tools}

(1) Social identity form:

The social identity of children is investigated by single topic selection. The paper adopts the single question: "you think you are: 1. Rural people. 2. Urban people. 3. Don't know," and through such a questionnaire, we investigate the status of urban migrant children on their own identity.

(2) Self-rating scale of mental health of middle school students in China:

The method used in this scale is five-point scoring, which is divided into 1-5 levels, each of which represents the following: from scratch, occasionally, sometimes, often, and always. It consists of 60 research projects and 10 factors, each of which contains 6 factors. The 10 factors were compulsive symptoms, depression, anxiety, paranoia, interpersonal tension and sensitivity, hostility, learning and stress, psychological disorder, poor adaptability, and out of control. Each factor reflects the following:

(1) Compulsive symptoms: these symptoms mainly show whether the subjects repeatedly check and count during the homework, always fear that their test results pass, and other unnecessary assumptions and unnecessary forced symptoms

(2) Depression: depression is mainly the subjects feeling monotonous about life, disappointment, and helplessness to their future and prone to negative emotional experience such as crying and psychological upset

(3) Anxiety: anxiety is mainly reflected in the subjects who often show emotional depression and feel very upset, resulting in poor sleep, psychological imbalance, and anger

(4) Paranoia: the main problems are feeling that others often say their own bad words behind the back, resulting in unwillingness to communicate with others, feeling that others are wrong in doing anything, others are always targeting themselves because they are foreign people, and they want to bully themselves

(5) Deal with tension and sensitivity in relationships: the main characteristics reflected by this factor are that the subjects generally think that others do not want to communicate with themselves and despise themselves, and they are always not understood, demanding and blaming others, 
believing that others are not friendly to themselves, and are easily hurt by them emotionally

(6) Hostility: the main feature is that the subjects cannot control their temper, and there is an impulse to smash things, which can easily cause the excitement and quarrel with others

(7) Study pressure: it shows that the subjects cannot adapt to the change of the learning mode and feel the difficulty of learning, the pressure is great, they are afraid of teachers, the situation of weariness of learning, the dislike of doing homework, and the fear and boredom of the test

(8) Psychological imbalance: the main performance is that the subjects think that teachers and parents will look at themselves differently, and they will feel that other children are better, often compare themselves with other students, and other problems

(9) Poor adaptation: the main problems of this factor are that the subjects cannot adapt to school life, teachers' teaching methods, and school environment, contact with more new things, and are unwilling to communicate with people and participate in other extracurricular activities

(10) Emotional imbalance: the emotional imbalance mainly refers to the subject's emotion which is easy to fluctuate and unstable, which is reflected in the study, which means that the students' attitude towards teachers, classmates, and parents is unstable and changeable

The main evaluation indexes of the mental health selfevaluation scale are as follows: The average score of 10 factors was below 2, indicating that the mental health was very good; the mental health status was mild in 2-2.99; in 3-3.99, it indicates that there are some problems in mental health status; between 4 and 4.99 , it indicates that mental health problems are serious and need to be dealt with as soon as possible; if it is 5 points, it means that mental health problems are very serious and should be paid great attention to.

3.2.3. Data Processing. Use SPSS 13.0 for data management and statistical analysis. First, use exploratory analysis to filter the suspicious data so that the complex data can be kept true. Second, analyze the variance of the remaining data, and visually compare the data.

3.2.4. Reliability of the Questionnaire. In order to test the reliability and stability of the questionnaire, firstly, the variance of the questionnaire results was calculated, and then the reliability of the returned questionnaire was tested by the method of "half-half reliability." Using formulas (6)-(8) to calculate the reliability coefficient, the correlation coefficient of the questionnaire is $q=0.883$. According to the theories and methods of modern scientific research, when the reliability of a test reaches 0.80 or more, it can be regarded as a test with higher reliability. The test results confirm that the questionnaire is reliable.

$$
\begin{aligned}
& C^{2}=\frac{\left(F-x_{1}\right)^{2}+\left(F-x_{2}\right)^{2}+\left(F-x_{3}\right)^{2}+\cdots+\left(F-x_{n}\right)^{2}}{n} \\
& q=1-\frac{C^{2}\left(1-q_{1}\right)}{C_{n}^{2}} \\
& q=\frac{2 q_{1}}{1+q_{1}}
\end{aligned}
$$

\section{Research Results and Discussion}

4.1. Differences in Social Identity. In order to better understand the real situation of urban migrant children in different grades, this paper analyzes the three levels of social identity of urban migrant children, and the results are shown in Table 1 and Figure 1: there are 65 junior high school students who think they are rural people, accounting for $32.34 \%$ of the total number of junior high school students in the survey, 41 junior high school students who think they are urban people, accounting for $20.4 \%$ of the total number of junior high school students in the survey, and 95 students with vague identification, accounting for $47.26 \%$ of the total number of junior high school students in the survey. There are 24 senior high school students who think they are rural people, accounting for $18.61 \%$ of the total number of senior high school students. There are 37 senior high school students who think they are urban people, accounting for $28.38 \%$ of the total number of senior high school students. There are 68 senior high school students who have vague identification, accounting for $53.1 \%$ of the total number of senior high school students.

It can be seen that the level of social identity of migrant children in different grades is different, as shown in Figure 1. The characteristics are as follows: the migrant children in the first grade of junior high school think that they are rural people more than those in the first grade of senior high school; that is, with the growth of children's age, this concept will decline. The migrant children in grade one are higher than those in grade one in urban identity; that is, with the growth of grade, the identity of urban people is on the rise. In terms of unclear identity, the migrant children in grade one of junior high school think that they are rural people, lower than those in senior high school.

4.2. Differences in Mental Health between Migrant Children and Urban Children. In order to study the difference in mental health between urban children and migrant children, the independent sample $t$-test method was used to compare the total average mental health scores and ten factors of urban migrant children and urban children. The results in Table 2 indicate the following: Migrant children and urban children have differences in emotional processing and interpersonal relationship processing, migrant children will show sensitivity, anxiety, paranoia, hostility, psychological imbalance, and learning pressure, but there is no significant statistical difference between the 
TABLE 1: Grade differences in social identity.

\begin{tabular}{lcccccc}
\hline & \multicolumn{2}{c}{ Rural identity } & \multicolumn{2}{c}{ City people identity } & \multicolumn{2}{c}{ Not clear } \\
& Frequency & Percentage & Frequency & Percentage & Frequency & Percentage \\
\hline First grade & 66 & 33.15 & 45 & 22.38 & 89 & 44.47 \\
High school & 27 & 20.77 & 33 & 25.38 & 70 & 53.85 \\
\hline
\end{tabular}

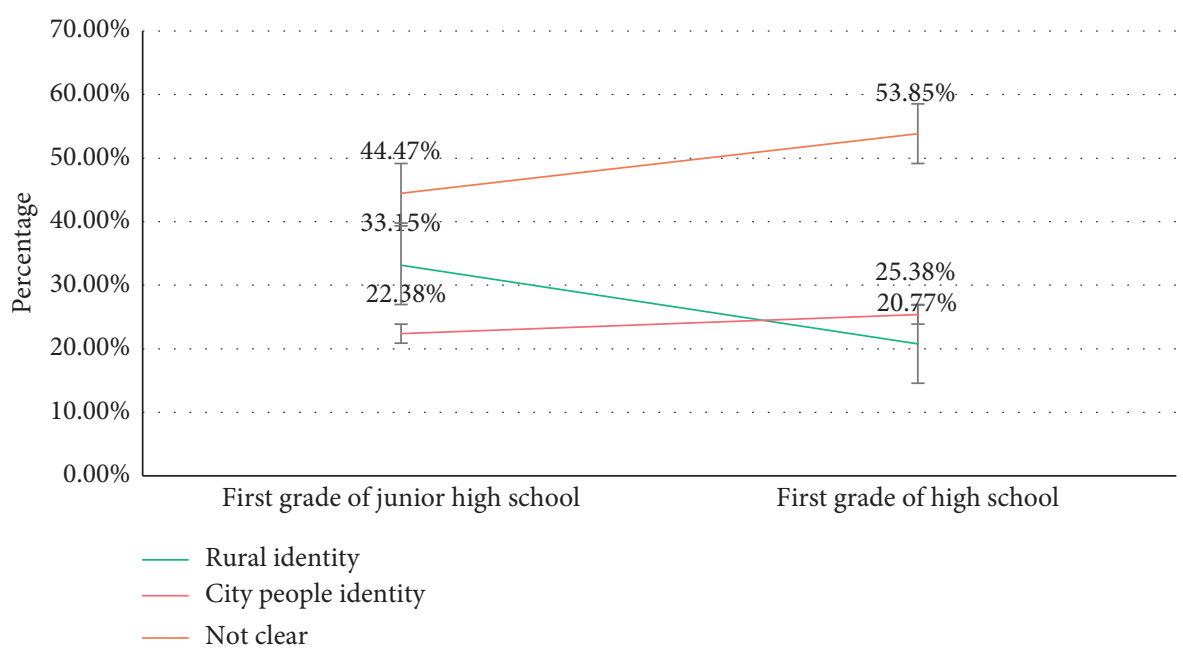

FIGURE 1: Grade differences in social identity.

total average score. It can be seen from Figure 2 that the total average score of migrant children in maladjustment, emotional imbalance, interpersonal tension and sensitivity, anxiety, paranoia, hostility, psychological imbalance, and learning pressure is higher than that of urban children, which also shows that the mental health of migrant children is lower than that of urban children to a certain extent, and the mental health of urban children is better.

\subsection{Analysis of Migrant Children's Mental Health}

(1) Lower self-esteem level:

In actual life, migrant children have no way to express their self-esteem clearly, but they will show a sense of loss when their self-esteem is hurt. With the growth of their age, it is possible that those acts of discrimination and hurting their self-esteem will make their body and mind suffer a great deal, leading to the increase of their sense of inferiority and the emergence of psychological problems. Migrant children, because they are still young and immature, tend to imagine themselves as they say, which is easy to confuse their own positioning and has a great impact on the correct self-cognition $[20,21]$. To sum up, self-esteem is the basic component of selfcognition to urban migrant children, which reflects a correct positioning and cognition of the individual. Selfesteem refers to the individual itself, which does not include the individual's sensory and evaluation of others, but may be affected by the evaluation from others. Selfesteem is an important form of the development of good mental health. People with strong self-esteem tend to show their own advantages and can naturally accept their own disadvantages. Maintaining high self-esteem is more conducive to the formation of good self-worth, and they can live a positive and enthusiastic life.

(2) Social maladjustment:

Migrant children move from the countryside to the city, especially in the first-tier cities such as Beijing and Shanghai. As the economic level and lifestyle of cities are very different from those of rural areas, differences in living environment will affect migrant children. They will affect them in a subtle way. When migrant children are deeply influenced by the material life of the city, they will imagine a real city person to live like that and learn to accept all this, and their thoughts will become urbanized. Due to the differences in culture, lifestyle, values, and so on, migrant children will need to adapt to these changes through self-regulation when they feel these changes and constantly change their behavior, ideas, and so on, so as to make them better adapt to the rhythm of the city. This process can be defined as the process of adapting to the society. When migrant children perceive the impact of urban culture, they may have a sense of frustration and inferiority, such as being excluded and loneliness rising. Then, they will have psychological discomfort, and their social adaptation will also be biased one after another.

(3) Sensitive discrimination:

From the perspective of the discriminated, discrimination is mainly a phenomenon produced by 
TABLe 2: Differences in mental health between migrant children and urban children.

\begin{tabular}{|c|c|c|c|c|}
\hline & $\begin{array}{c}\text { Migrant children } \\
M \pm \mathrm{SD}\end{array}$ & $\begin{array}{c}\text { City children } \\
M \pm \mathrm{SD}\end{array}$ & $t$ & $p$ \\
\hline Maladaptation & $1.97 \pm 0.63$ & $1.86 \pm 0.57$ & 1.932 & 0.057 \\
\hline Emotional imbalance & $2.09 \pm 0.66$ & $2.05 \pm 0.75$ & 0.568 & 0.571 \\
\hline Obsessive-compulsive symptoms & $2.02 \pm 0.58$ & $2.04 \pm 0.61$ & -1.031 & 0.312 \\
\hline Interpersonal tension and sensitivity & $1.91 \pm 0.61$ & $1.85 \pm 0.63$ & 1.049 & 0.301 \\
\hline Depression & $1.82 \pm 0.62$ & $1.83 \pm 0.63$ & -0.198 & 0.837 \\
\hline Anxiety & $1.92 \pm 0.76$ & $1.91 \pm 0.73$ & 0.382 & 0.707 \\
\hline Paranoia & $1.83 \pm 0.65$ & $1.82 \pm 0.61$ & 0.601 & 0.562 \\
\hline Hostility & $1.77 \pm 0.67$ & $1.76 \pm 0.68$ & 0.468 & 0.641 \\
\hline Psychological imbalance & $1.73 \pm 0.64$ & $1.69 \pm 0.59$ & 0.858 & 0.387 \\
\hline Study-induced stress & $1.91 \pm 0.51$ & $2.12 \pm 0.82$ & 0.049 & 0.958 \\
\hline
\end{tabular}

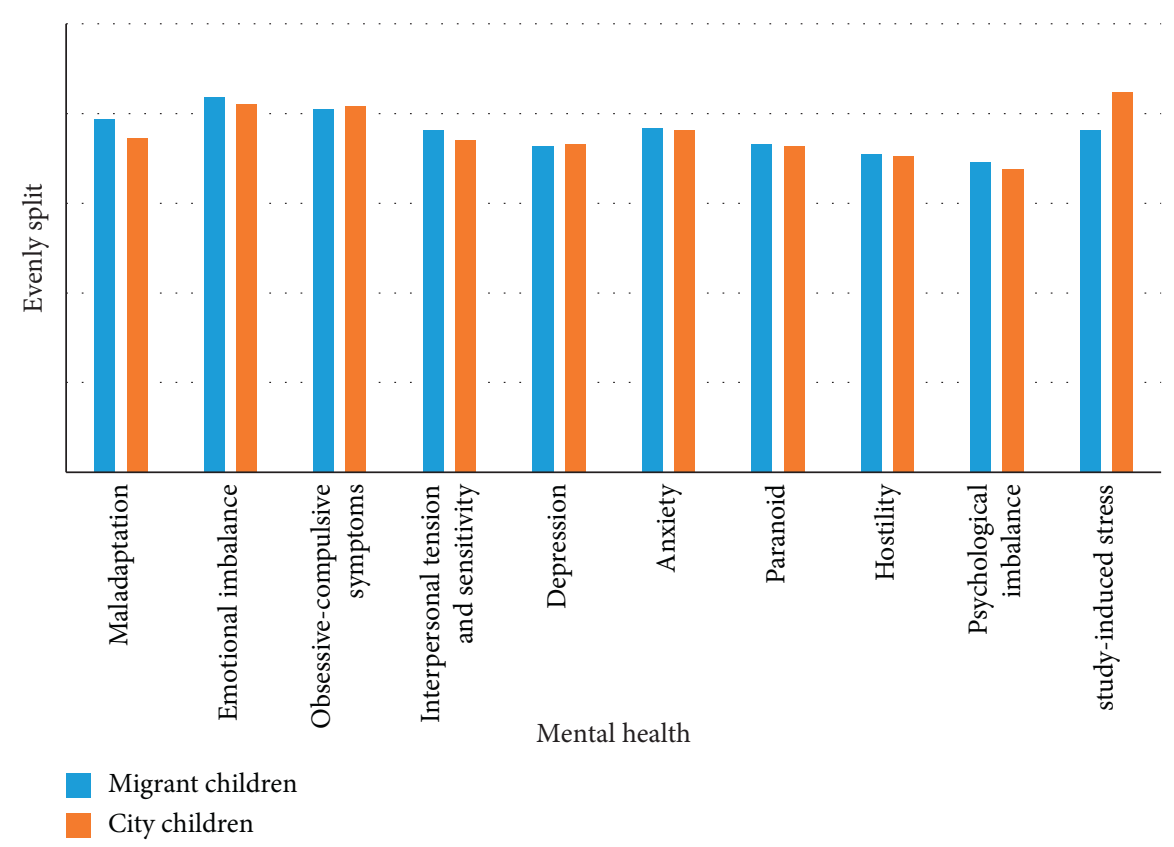

Figure 2: Differences in mental health between migrant children and urban children.

perception. The discriminated can obviously feel the negative impact and the harm brought by discrimination. It is very unfair to treat a person with this perception of discrimination. It is a very unreasonable social system. The manifestation of discrimination may be expressed by actual action, which is very harmful to a person. If urban migrant children encounter discrimination or exclusion, it will cause great harm to them physically and mentally.

\section{Conclusions}

After the above research, we can draw the following conclusions. From the questionnaire survey, it is found that the problem of unclear social identity of migrant children in this city is universal and ambiguous. There is no significant difference in the ten factors of mental health between urban migrant children and urban children. However, urban migrant children will have sensitivity, anxiety, paranoia, hostility, psychological imbalance, learning pressure, and other problems in adaptability, emotional processing, and interpersonal relationship, and the total average score will be greater than that of urban children, which indicates that the mental health of urban children is better than that of migrant children. We need to help migrant children adapt to the new environment from the heart and accept the new environment, including new culture, new customs, new campus, and new life. Only when they accept the new environment from the heart can they better adapt to the environment and develop better in the city.

\section{Data Availability}

Data sharing is not applicable to this article as no datasets were generated or analysed during the current study.

\section{Conflicts of Interest}

The author declares that there are no conflicts of interest. 


\section{Acknowledgments}

This paper is the periodic research results of "Research on the Construction of Relief System for Difficult Children in Shandong Province from the Concept of Multiple Cogovernance" (project no. 2020VC12028), a Key Scientific Research Project of Colleges and Universities in Shandong Province in 2020.

\section{References}

[1] A. Umer, B. Nazir, and Z. Ahmad, "Adaptive market-oriented combinatorial double auction resource allocation model in cloud computing," The Journal of Supercomputing, vol. 16, no. 1, pp. 1-11, 2021.

[2] G. Baranwal and D. P. Vidyarthi, "A truthful and fair multiattribute combinatorial reverse auction for resource procurement in cloud computing," IEEE Transactions on Services Computing, vol. 12, no. 6, pp. 851-864, 2019.

[3] L. Ban, S. Guo, R. W. Scherpbier, X. Wang, H. Zhou, and L. J. Tata, "Child feeding and stunting prevalence in leftbehind children: a descriptive analysis of data from a central and western Chinese population," International Journal of Public Health, vol. 62, no. 1, pp. 143-151, 2017.

[4] C. Lämmle, O. Wartha, S. Kobel, T. Wirt, A. Kelso, and C. Kutzner, "Intervention effects of the school-based health promotion program "join the healthy boat" on motor abilities of children with migration background," Health, vol. 09, no. 3 , pp. 520-533, 2017.

[5] C. W. Pan, B. Shi, H. Zhong, J. Li, and Q. Chen, "The impact of parental rural-to-urban migration on children's refractive error in rural China: a propensity score matching analysis," Ophthalmic Epidemiology, vol. 27, no. 1, pp. 1-6, 2019.

[6] T. Liu, K. Holmes, and J. Albright, "Urban teachers' perceptions of inclusion of migrant children in the Chinese educational institution: a comparative study," International Journal of Inclusive Education, vol. 19, no. 9, pp. 1-15, 2016.

[7] J. Fang, "Research on the family language education for migrant children in northwest China: based on the sample of xi'an city," Educational Sciences: Theory and Practice, vol. 18, no. 5, 2018.

[8] K. Antia, J. Boucsein, A. Deckert, P. Dambach, J. Račaitè, and G. Šurkiene, "Effects of international labour migration on the mental health and well-being of left-behind children: a systematic literature review," International Journal of Environmental Research and Public Health, vol. 17, p. 4335, 2020.

[9] A. G. Larsen, "Protection or Participation?: editorial evaluation of two news serials concerning irregular migrant children," Journalism Practice, vol. 11, no. 7, pp. 1-16, 2016.

[10] Q.-F. Dong, D.-K. Chen, and T. Wang, "Urban community structure detection based on the OD of traffic analysis zones," Modern Physics Letters B, vol. 33, no. 13, Article ID 1950164, 2019.

[11] G. Baranwal and D. P. Vidyarthi, "A fair multi-attribute combinatorial double auction model for resource allocation in cloud computing," Journal of Systems and Software, vol. 108, pp. 60-76, 2015.

[12] A. B. Zaslavsky, C. Perera, and D. Georgakopoulos, Sensing as a Service and Big Data in International Conference on Advances in Cloud Computingp. 219, ACC-2012, Bangalore, India, 2012.

[13] S. R. Chandra and Y. Wang, "Cloud things construction-the integration of internet of things and cloud computing," Future
Generation Computer Systems, vol. 56, no. C, pp. 684-700, 2016.

[14] F. Liang, Y. Quan, A. Wu et al., "Insulin-resistance and depression cohort data mining to identify nutraceutical related DNA methylation biomarker for type 2 diabetes," Genes and Diseases, vol. 8, no. 5, pp. 669-676, 2021.

[15] S. He, D. Luo, and K. Guo, "Analysis of factors affecting the coordinated development of urbanization and the ecological resource environment in southwest China based on data mining," Journal of Urban Planning and Development, vol. 147, no. 3, pp. 71-88, 2021.

[16] Z. Wang, X. Lin, and X. Hou, "Urban adaptation of migrant children and influencing factors-an overview of research in the past 20 Years," Journal of Beijing Normal University: Social Science Edition, vol. 2, pp. 37-46, 2016.

[17] Y. Zhang, A. Du, and Y. Zhou, "The relationship between migrant children's perception of discrimination and urban adaptation: the multiple mediating effects of social support and identity integration," China Special Education, vol. 8, pp. 55-60, 2017.

[18] T. H. Sardar and A. Anrisa, "An analysis of map reduce efficiency in document clustering using parallel K-means algorithm," Future Computing and Informatics Journal, vol. 3, no. 2, pp. 200-209, 2018.

[19] K. Mostafa, A. Attallab, and T. Hegazy, "Data mining of school inspection reports to identify the assets with top renewal priority," Journal of Building Engineering, vol. 41, no. 3, pp. 99-111, 2021.

[20] G. N. Lima and M. Lombardo, "Urban climatology in Brazil: an analysis based on the methodology of the urban climate system," Environment Conservation Journal, vol. 20, no. 1\&2, pp. 1-8, 2019.

[21] J. Gartner, D. B. Larson, and G. D. Allen, "Religious commitment and mental health: a review of the empirical literature," Journal of Psychology \& Theology, vol. 19, no. 1, pp. 6-25, 2018. 